\title{
7 Schlussfolgerung und Ausblick
}

Anhand der in der vorliegenden Arbeit durchgeführten Sensitivitätsanalyse werden Einflussmöglichkeiten aufgezeigt, das Lenkungsverhalten im OnCenter Bereich zu beeinflussen. So wirken sich beispielsweise klassische konstruktive Lenkungs- und Fahrzeugparameter wie die Lenkübersetzung oder die Achssteifigkeit auf die Kennwerte Lenkungssteifigkeit, Lenkradmomententotband, Lenkungsempfindlichkeit und Lenkungsansprechen aus. Das Potential von elektrisch unterstützten Lenksystemen liegt u.a. darin, weitere Einflussmöglichkeiten auf die Lenkungseigenschaften $\mathrm{zu}$ bieten. Eine Variation der Handmomentenunterstützung beeinflusst die Kennwerte Lenkradmomentgradient und Lenkradmoment bei $0 \mathrm{~m} / \mathrm{s}^{2}$ und bei $1 \mathrm{~m} / \mathrm{s}^{2}$. Durch die Funktion aktiver Rücklauf kann ebenfalls Einfluss auf die Kennwerte Lenkradmomentgradient, Lenkradmomententoband und darüber hinaus auch auf den Kennwert Lenkradmomentgradient genommen werden.

Wie exemplarisch aufgezeigt wurde, lässt sich mit einer Parametervariation die subjektive Wahrnehmung des Fahrzeugs z. B. hinsichtlich Sportlichkeit oder Komfort beeinflussen. Somit erweitern sich die Abstimmungsmöglichkeiten für elektrisch unterstützte Lenksysteme im Vergleich zu hydraulisch unterstützten Systemen und lassen eine weitergehende Individualisierung zu.

Um gefundene Abweichungen zwischen Komponentenprüfstands- und Gesamtfahrzeugmessungen weitergehend $\mathrm{zu}$ analysieren, sollte in künftigen Untersuchungen das Lenksystem auf einem Prüfstand untersucht werden, der auch das Schwingungsverhalten des Fahrzeugs nachbildet. Ein solcher Prüfstand befindet sich am IVK der Universität Stuttgart gerade im Aufbau, stand jedoch für die Messungen für diese Arbeit noch nicht zur Verfügung.

Des Weiteren kann Ziel weiterführender Arbeiten sein, das erstellte Modell in einem Fahrsimulator mit Probanden zum Einsatz zu bringen, um die subjektive Bewertung der Änderung der Parameter genauer zu untersuchen. 